\title{
Neutron interferometry, fifth force and axion like particles
}

\author{
A. Capolupo ${ }^{1, \mathrm{a}}$, S. M. Giampaolo ${ }^{2, \mathrm{~b}}$, A. Quaranta ${ }^{1, \mathrm{c}}$ \\ ${ }^{1}$ Dipartimento di Fisica "E.R. Caianiello", Università di Salerno, and INFN-Gruppo Collegato di Salerno, Via Giovanni Paolo II, 132,84084 \\ Fisciano, SA, Italy \\ ${ }^{2}$ Institut Ruder Bošković, Bijenička cesta 54, 10000 Zagreb, Croatia
}

Received: 31 August 2021 / Accepted: 27 November 2021 / Published online: 18 December 2021

(C) The Author(s) 2021

\begin{abstract}
We propose a new possible detection strategy to reveal the fermion-fermion interaction mediated by axions and axion-like particles, based on interferometric measurement of neutron beams. We consider an interferometer in which the neutron beam is split in two sub-beams propagating in regions with differently oriented magnetic fields. The beam paths and the strength of the magnetic fields are set in such a way that the phase difference depends only on the axion-induced interaction. The resulting phase difference is directly related to the presence of axions. Our results show that such a phase might represent, in the future, a tool to probe the existence of axions and axion-like particles or a fifth force with interferometry.
\end{abstract}

\section{Introduction}

The need for physics beyond the standard model of particles [1] is evident in several phenomena, from neutrino mixing [2-5] to the dark sector of the universe [6,7]. A hint of new physics also comes from strong interactions, for which the standard model allows an arbitrary CP symmetry violation, through the $\theta$ term, that is not observed experimentally. This tension, known as the strong $\mathrm{CP}$ problem, can be elegantly reconciled by promoting the $\theta$ parameter to a dynamical field, as originally proposed by Peccei and Quinn $[8,9]$. The Peccei-Quinn mechanism gives rise to pseudo-scalar particles known as axions [8-12] which hold a number of interesting properties. In particular, they soon gained attention as a possible dark matter component [13] in virtue of their tiny interactions with ordinary particles. Inspired by the PecceiQuinn model, a considerable variety of axion-like particles (ALPs) models has been proposed, including the DSFZ [1416] and the KSVZ axions [16-18], with masses ranging

\footnotetext{
a e-mail: capolupo@sa.infn.it

be-mail: sgiampa@irb.hr

c e-mail: anquaranta@unisa.it (corresponding author)
}

everywhere from the ultralight $[19,20] m \simeq 10^{-22} \mathrm{eV}$ to the heavy axions [21] $m \simeq 1 \mathrm{TeV}$. This proliferation of models is justified, at least partially, by the primary role that ALPs would play in cosmology. Indeed, ALPs represent to date one of the most convincing particle explanation for dark matter and are actively searched for in several experiments [22-30]. Nonetheless, up to now no evidence for ALPs has been found.

Besides the interaction with the electromagnetic field, on which the vast majority of the ongoing experiments is based, ALPs are expected to experience an interaction with fermionic fields. The latter yields an effective fermionfermion interaction $[31,32]$ in which the ALP plays the role of a mediating boson. For this reason, the detection of ALPs is naturally tied not only to electrodynamics but also to the possibility of experimentally probing interactions between fermions [33-36]. On the other hand, in the context of neutron physics, interferometry has proven to be a formidable tool in investigating particle interactions and their quantum properties. Neutron interferometry is indeed a subject of study in its own right [37] and has granted us the chance to verify many theoretical predictions. These include the Sagnac effect [38], the geometric phase [39-41], wave-particle duality and the change of sign in the spin $1 / 2$ wave-function after a $2 \pi$ rotation [37]. Moreover, experiments oriented to searches for non-Newtonian gravity at short distances and the observation of relativistic proper time effects, related to a gravitational redshift, could represent new topics to be analyzed with neutron interferometry [37].

The purpose of this paper is to take advantage of the versatility of neutron interferometry in order to suggest an alternative approach to the detection of ALPs for future experiments. We show that the axion-induced interactions between neutrons sum up to give an additional magnetic term, whose presence affects the phase of the neutrons and produces an extra phase difference in interferometric experiments. We consider an idealized interferometric setup in which a collimated neutron beam is split into two sub-beams that are later 
let interfere with each other. Each of the sub-beams is subject to an external magnetic field of equal strength but different direction. In particular, if one of the two magnetic fields is set in the direction of propagation of the relative sub-beam and the other is orthogonal to the propagation, the neutrons will gain a path-dependent phase factor that can be easily detected by an interferometer. The experimental parameters are fixed in such a way that the interference pattern is only due to the magnetic and axion-induced interactions. We show how to isolate the axion contribution by appropriately choosing the neutron path and we set an arm length such that the contribution to the phase difference given by the dipole-dipole interaction is an integer multiple of $2 \pi$. In this way, we obtain a phase difference depending only on the axion-mediated interaction. We discuss how the presence of ALPs might be tested with interferometry in a significant portion of parameter space. We consider an oversimplified setting to illustrate the basic idea. In a forthcoming paper we will consider different geometries and a more realistic setup.

The paper is structured as follows. In Sect. 2, we present the neutron-neutron interaction mediated by axions and introduce the two-neutron interaction Hamiltonian, containing the dipole-dipole and the axion-mediated interaction. In Sect. 3, we generalize the neutron interaction to the case of an arbitrary number of neutrons, obtaining an effective one particle Hamiltonian for the interacting neutrons. In Sect. 4 we show the setup for the neutron interferometry, the evolution of the neutron state, the expression of the effective magnetic fields and the expressions of the phase differences between the two beams at the interference plane. A numerical analysis is presented in Sect. 5. Discussions and conclusions are reported in Sect. 6.

\section{Neutron-neutron interactions}

To begin, let us briefly resume the neutron-neutron interaction due to axion exchange. For two generic fermion fields, the interaction Lagrangian is given by $[31,32]$

$\mathcal{L}_{I N T}=-\sum_{j=1,2} i g_{a j} \phi \bar{\psi}_{j} \gamma_{5} \psi_{j}$

where $\phi$ is the axion field, $\psi_{1}, \psi_{2}$ are the fermion fields and $g_{a j}$ are the (dimensionless) effective axion-fermion coupling constants, which depend on the fermions considered and the axion (or ALP) model. The couplings are generally small $g_{a j} \ll 1$, allowing a perturbative treatment of the interaction. For two neutrons, only one term is present in Eq. (1), with $g_{a j} \equiv g_{a N N}$ the axion-neutron coupling. Compared to the more familiar axion-photon coupling $g_{a \gamma \gamma}$, which for many axion models is proportional to the axion mass $m$, the axion-neutron coupling $g_{a N N}$ is much less constrained experimentally and depends critically on the underlying ALP model. For instance, the coupling of axions to nucleons is proportional to the axion mass for the DFSZ model, but vanishes for hadronic axions [16]. We mention that currently the strongest bounds on the coupling of axions to neutrons come from the astrophysical analyses [42-44]. These bounds are however subject to many uncertainties and depend strongly on the models considered. Since we wish to provide a modelindependent analysis of the axion-neutron interactions, we shall not assume a specific relation between coupling and mass, and rely on model-independent constraints in order to select a sensible range of couplings. It can be shown that in the non-relativistic limit the Lagrangian of Eq. (1) gives rise to a two-body potential given by [32]

$$
\begin{aligned}
H_{p}(\mathbf{r})= & -\frac{g_{a N N}^{2}}{4 \pi M^{2}} e^{-m r}\left[\left(\sigma_{\mathbf{1}} \cdot \sigma_{\mathbf{2}}\right)\left(\frac{m}{r^{2}}+\frac{1}{r^{3}}\right)\right. \\
& \left.-\left(\sigma_{\mathbf{1}} \cdot \hat{\boldsymbol{r}}\right)\left(\sigma_{\mathbf{2}} \cdot \hat{\boldsymbol{r}}\right)\left(\frac{m^{2}}{r}+\frac{3 m}{r^{2}}+\frac{3}{r^{3}}\right)\right],
\end{aligned}
$$

where $M \simeq 939 \mathrm{MeV}$ is the mass of the neutron [16], $m$ is the mass of the ALP, $r$ is the relative position of the two neutrons, $r$ its modulus and $\sigma_{j}$ are the spin operators of the $j$ th neutron. In Eq. (2) we have dropped a term proportional to the Dirac delta $\delta^{3}(\boldsymbol{r})$ assuming a non vanishing inter-neutron distance $r \neq 0$.

The Hamiltonian (2) describes the interaction between two neutrons mediated by axions. Notice that an interaction of the kind of Eq. (2) corresponds to a long-range spin-dependent force among the neutrons. Therefore our analysis well fits within the context of fifth force experiments [33-36,45,46]. In the following we shall assume that the neutron velocities are non-relativistic and analyze the interaction Hamiltonian (2) within the context of ordinary quantum mechanics. The state of the single neutron shall be taken as the product of a spatial and a spin wavefunction. Since we are interested in the evolution of the neutron state, it is our duty to write down the appropriate Schroedinger equation. To this end we need to take into account all the possible interactions between neutrons. The gravitational interaction is easily seen to be irrelevant, due to the smallness of the masses involved. In addition, we shall always assume a relative distance $r>10^{-12} \mathrm{~m}$, so that the short-range nuclear interactions can also be ignored. With this assumption the only other relevant interaction is the magnetic one between the neutron dipoles. The two-neutron interaction Hamiltonian, comprising the magnetic and the axion-mediated interaction can thus be written as

$$
\begin{aligned}
H= & -\frac{\mathcal{A}}{r^{3}}\left[\left(3-\mathcal{B} e^{-m r} K^{(a)}(r)\right) \sigma_{1}^{r} \sigma_{2}^{r}\right. \\
& \left.-\left(1-\mathcal{B} e^{-m r} K^{(b)}(r)\right) \boldsymbol{\sigma}_{1} \cdot \boldsymbol{\sigma}_{2}\right] .
\end{aligned}
$$

where the parameter $\mathcal{A}=\frac{g^{2} \alpha}{16 M^{2}}$ is the strength of the magnetic interaction ( $g$ is the neutron g-factor), $\mathcal{B}=\frac{g_{a N N}^{2}}{\pi \alpha g^{2}}$ is the 
relative weight of the axion interaction and $\alpha \simeq \frac{1}{137}$ denotes the fine-structure constant. Here the dimensionless (in natural units $c=1=\hbar$ ) functions $K^{(\lambda)}(r)$ (with $\lambda=a, b$ ) are defined as

$$
\begin{aligned}
& K^{(a)}(r)=m^{2} r^{2}+3 m r+3, \\
& K^{(b)}(r)=m r+1,
\end{aligned}
$$

The operator $\sigma^{r}$ is defined as $\sigma_{i}^{r}=\boldsymbol{\sigma}_{i} \cdot \hat{\boldsymbol{r}}$, with $\hat{\boldsymbol{r}}$ the unit vector along the direction joining the two neutrons.

\section{The effective magnetic field}

The two-neutron interaction can easily be generalized to an arbitrary number of neutrons. Our starting point is the total two-body Hamiltonian of Eq. (3), with a notation suitable for an arbitrary number of neutrons:

$$
\begin{aligned}
H_{i j}= & -\frac{\mathcal{A}}{r_{i j}^{3}}\left[\left(3-\mathcal{B} e^{-m r_{i j}} K^{(a)}\left(r_{i j}\right)\right) \sigma_{i}^{r_{i j}} \sigma_{j}^{r_{i j}}\right. \\
& \left.-\left(1-\mathcal{B} e^{-m r_{i j}} K^{(b)}\left(r_{i j}\right)\right) \boldsymbol{\sigma}_{i} \cdot \boldsymbol{\sigma}_{j}\right],
\end{aligned}
$$

where the $K^{\lambda}$ functions have been defined in Eqs. (4) and (5). In the case of two particles it is natural to choose one of the spatial axes as to coincide with the line joining the two $\hat{\boldsymbol{r}}$. For a generic number of particles there are many such directions $\hat{\boldsymbol{r}}_{i j}$, one for each pair, and thus one has to express Eq. (6) with respect to an arbitrary, but fixed, spatial triple $\hat{\boldsymbol{x}}, \hat{\boldsymbol{y}}, \hat{z}$. Hence, for each pair $i, j$, we introduce the projections $R_{i j}^{u}=\hat{\boldsymbol{r}}_{i j} \cdot \hat{\boldsymbol{u}}$, with $u=x, y, z$. In terms of the polar angles $\theta_{i j}, \phi_{i j}$, the projections can explicitly be written as $R_{i j}^{x}=$ $\sin \left(\theta_{i j}\right) \cos \left(\phi_{i j}\right), R_{i j}^{y}=\sin \left(\theta_{i j}\right) \sin \left(\phi_{i j}\right)$ and $R_{i j}^{z}=\sin \left(\theta_{i j}\right)$. The operators $\sigma_{i}^{r}$ are then obviously equal to

$\sigma_{i}^{r_{i j}}=R_{i j}^{x} \sigma_{i}^{x}+R_{i j}^{y} \sigma_{i}^{y}+R_{i j}^{z} \sigma_{i}^{z}$.

The notation $\sigma_{i}^{r_{i j}}$ is used to remark that while these operators act only upon the space of the $i$ th particle, their form depends on the specific pair $i, j$ considered. Writing the projections explicitly, Eq. (6) becomes

$$
\begin{aligned}
H_{i j}= & -\frac{\mathcal{A}}{r_{i j}^{3}}\left(1-\mathcal{B} e^{-m r_{i j}} K^{(b)}\left(r_{i j}\right)\right) \sigma_{i} \cdot \sigma_{j} \\
& -\frac{\mathcal{A}}{r_{i j}^{3}}\left(3-\mathcal{B} e^{-m r_{i j}} K^{(a)}\left(r_{i j}\right)\right) \\
& \times\left[\left(R_{i j}^{x}\right)^{2} \sigma_{i}^{x} \sigma_{j}^{x}+\left(R_{i j}^{y}\right)^{2} \sigma_{i}^{y} \sigma_{j}^{y}+\left(R_{i j}^{z}\right)^{2} \sigma_{i}^{z} \sigma_{j}^{z}\right. \\
& +R_{i j}^{x} R_{i j}^{y}\left(\sigma_{i}^{x} \sigma_{j}^{y}+\sigma_{i}^{y} \sigma_{j}^{x}\right)+R_{i j}^{x} R_{i j}^{z}\left(\sigma_{i}^{x} \sigma_{j}^{z}+\sigma_{i}^{z} \sigma_{j}^{x}\right) \\
& \left.+R_{i j}^{y} R_{i j}^{z}\left(\sigma_{i}^{y} \sigma_{j}^{z}+\sigma_{i}^{z} \sigma_{j}^{y}\right)\right] .
\end{aligned}
$$

Then, the total interaction Hamiltonian is simply

$H=\frac{1}{2} \sum_{i, j} H_{i j}$

where the sum runs over all the neutron pairs $i \neq j$ and the factor $\frac{1}{2}$ accounts for double counting.

To go further, we express (8) in a more compact way. Setting $C(\boldsymbol{r})$ and $D(\boldsymbol{r})$ as

$C(\boldsymbol{r})=\frac{\mathcal{A}}{r^{3}}\left(1-\mathcal{B} e^{-m r} K^{(b)}(r)\right)$,
$D(\boldsymbol{r})=\frac{\mathcal{A}}{r^{3}}\left(3-\mathcal{B} e^{-m r} K^{(a)}(r)\right)$,

we can introduce the symmetric matrix $K^{u v}(\boldsymbol{r})$

$K^{u v}(\boldsymbol{r})=C(\boldsymbol{r}) \delta^{u v}-D(\boldsymbol{r}) R^{u}(\boldsymbol{r}) R^{v}(\boldsymbol{r})$.

where $u, v=x, y, z$. Here $\delta^{u v}$ is the Kronecker symbol and $R^{u}(\boldsymbol{r})=\hat{\boldsymbol{r}} \cdot \hat{\boldsymbol{u}}$ (notice that $R_{i j}^{u}=R^{u}\left(\boldsymbol{r}_{j}-\boldsymbol{r}_{i}\right)$ ). The interaction Hamiltonian can then be written as

$H=\frac{1}{2} \sum_{i \neq j} \sum_{u, v} K^{u v}\left(\boldsymbol{r}_{j}-\boldsymbol{r}_{i}\right) \sigma_{i}^{u} \sigma_{j}^{v}$.

From Eq. (13) it is easy to see that the analysis of the evolution of the $n$-neutron state with a generic $n$, interacting via $H$ is in principle a complicated many-body problem, which hardly admits an analytical treatment if no further assumptions are made. In the present context, however, we are not interested in correlations and collective effects, and are concerned only with the evolution of the single neutron state. For this reason it is convenient to encode the interaction with all the other nucleons in an effective one-particle potential, by resorting to a mean field approach. Given a neutron at position $\boldsymbol{r}_{i}$ and Pauli operator $\boldsymbol{\sigma}_{i}$, the instantaneous interaction Hamiltonian due to the other neutrons is $H_{i}=\sum_{u, v} \sum_{j \neq i} K^{u v}\left(\boldsymbol{r}_{i j}\right) \sigma_{j}^{u} \sigma_{i}^{v}$, where the sum runs over all the other neutrons $j$. To obtain an effective local potential for the single neutron, this equation is replaced with its expectation value on the state of the other nucleons. In doing so, the single particle Hamiltonian $H$ acquires a very neat interpretation in terms of an effective magnetic field due to the other neutrons. Indeed, setting

$\mu \boldsymbol{B}_{i}\left(\boldsymbol{r}_{i}\right)=-\sum_{u} \sum_{j} K^{u v}\left(\boldsymbol{r}_{i j}\right)\left\langle\sigma_{j}^{u}\right\rangle$,

we recover the usual term of a spin interacting with a magnetic field $H_{i}=-\mu\left(\boldsymbol{B}_{i}\left(\boldsymbol{r}_{i}\right)\right) \cdot \boldsymbol{\sigma}$. Once the effective magnetic field is computed for a particular spatial-spin configuration, one simply plugs the one-particle operator in the Schroedinger equation in order to study the evolution of the single neutron state. 


\section{Neutron interferometry and ALPS}

In the foregoing we shall consider collimated neutron beams. For cold neutrons, collimation and a small beam width of the order of $10 \mu \mathrm{m}$ can be obtained by any of the means described in Ref. [47], i.e. by use of collimation slits, reflected beams or neutron waveguides. The neutron flux and its intensity depend on the neutron source (typical intensities at the detector are in the range $I=10^{4}-10^{8} \mathrm{n} / \mathrm{s}$ ) and, of course, on the collimation device, which cuts off a part of the incoming neutron flux. We will also assume that only neutrons around a given value of the kinetic energy $K$ are selected, for instance by use of a monochromator. In its trip from the collimation device to the detector, the neutron beam experiences an angular spread whose entity depends on the collimation device and on the collimator-detector distance. Very small angle spreads can be obtained for reflected beams [47]. We can generally consider that the beam is distributed with cylindrical symmetry around the beam axis $\hat{\boldsymbol{y}}$, and, assuming that it is sufficiently thin, we model the beam as a monodimensional system. As a first instance, we also neglect the losses due to propagation and consider the beam intensity as a constant.

\subsection{The setup}

Given these preliminaries, we now discuss an idealized setup aimed at revealing the axion-mediated interaction among neutrons. A beam of cold or ultra-cold neutrons, whose source SRC might be an appropriate reactor, is conveyed to an external apparatus EXT which has the purpose of rendering the beam as monochromatic as possible (e.g. using monochromators) and also serves as a collimator, making the beam as thin and linear as possible. The beam then goes through a beam splitter BS which splits the beam into two sub-beams $I$ and $I I$. Then each of the sub-beams enters a Stern-Gerlach like apparatus that selects two different spin polarizations $\boldsymbol{P}_{I}$ and $\boldsymbol{P}_{I I}$ (the upper case indices $J=I, I I$ and roman numerals are used to denote the specific subbeam). The average polarizations are maintained by two constant and uniform magnetic fields $\boldsymbol{B}_{I}^{0}$ and $\boldsymbol{B}_{I I}^{0}$ surrounding the sub-beams $I$ and $I I$ of the same strength but with distinct direction, i.e. $\boldsymbol{B}_{J}^{0}=B_{0} \boldsymbol{P}_{J}$.

The setup is schematically pictured in Fig. 1 . The fractional intensities of the subbeams with respect to the initial beam $I_{0}$, namely $\chi_{I}=\frac{I_{I}}{I_{0}}$ and $\chi_{I I}=\frac{I_{I I}}{I_{0}}$ should be as close as possible $\chi_{I} \simeq \chi_{I I}$. The intensities regulate the average distance between successive neutrons in the two subbeams $d_{I}$ and $d_{I I}$, in the following way. Assuming a perfectly linear beam and an average distance $d$, in a time $d t$ the number of neutrons passing through a given point on the line of propagation $d N$ is evidently

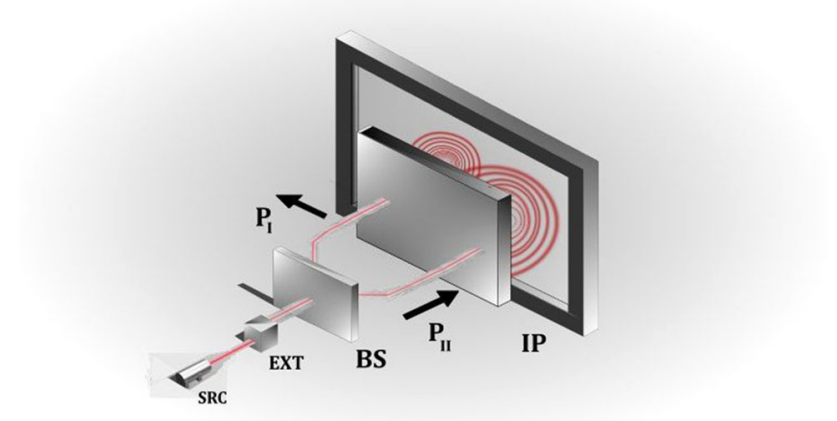

Fig. 1 Schematic diagram of the interferometric apparatus. The beam from the neutron source SRC is conveyed to an external apparatus EXT consisting of a collimation device and a monochromator. The collimated and monochromatic beam is then conveyed to a beam splitter BS which splits the beam into two subbeams with different average spin polarizations $\boldsymbol{P}_{I}$ and $\boldsymbol{P}_{I I}$. Finally the two subbeams interfere at the interference plane IP

$d N=\frac{\bar{v} d t}{d}$

where $\bar{v}$ is the average neutron velocity in the direction of propagation. Then

$\frac{d N}{d t}=I=\frac{\bar{v}}{d}$

which of course means that the higher is the intensity, the smaller is the average distance between two consecutive neutrons. Therefore $d_{I}=\frac{\bar{v}}{I_{I}}$ and $d_{I I}=\frac{\bar{v}}{I_{I I}}$ (where we have assumed the same average velocity in both subbeams). Considering constant intensities $I_{J}$ and constant average neutron velocities $\bar{v}_{J}$, the average distances themselves $d_{J}$ are constant, resulting in a time-independent one particle Hamiltonian $H$, and if $\chi_{I} \simeq \chi_{I I}, d_{I} \simeq d_{I I}$. The $d_{J}$ should be in any case large enough to neglect the effects of nuclear interactions $d_{J}>10^{-12} \mathrm{~m}$. For simplicity, from now on, we assume that all the nearest neighbour distances within sub-beam $J$ are constant and equal to $d_{J}$.

The two polarized sub-beams then go through two optical paths of the same length to the interference plane IP, where the interference pattern is observed.

\subsection{Evaluation of effective magnetic fields}

Let $\hat{\boldsymbol{y}}_{J}$ denote the direction of propagation in sub-beam $J$. Choosing $\boldsymbol{P}_{I}$ to be orthogonal to $\hat{\boldsymbol{y}}_{I}$ and $\boldsymbol{P}_{I I}$ parallel to $\hat{\boldsymbol{y}}_{I I}$, from Eq. (14), we can find the expression of the magnetic field acting on a single neutron spin. Indeed, accordingly with our scheme, the two sub-beams can be approximated as a one-dimensional regular chain in which the distance between neighboring neutrons is constant and equal to $d_{J}$. Then, for each neutron, limiting the sum to the closest $2 n$ neighboring neutrons, the effective magnetic fields in the two sub-beams 
become

$$
\begin{aligned}
(\mu \boldsymbol{B})_{n I} & =\sum_{l=1}^{n}\left[-2 C\left(l d_{I}\right) \boldsymbol{P}_{I}\right] \\
(\mu \boldsymbol{B})_{n I I} & =\sum_{l=1}^{n} 2\left[D\left(l d_{I I}\right)-C\left(l d_{I I}\right)\right] \boldsymbol{P}_{I I} .
\end{aligned}
$$

From Eqs. (10) and (11) we see that each term in the two sums that define the magnetic fields are porportional to $d^{-3}$. This implies that the sums in Eqs. (17) and (18) converge rapidly to their limiting value $(n \rightarrow \infty)$, as neutrons farther away give smaller and smaller contributions. Therefore, in the evaluation of the magnetic fields, we can consider the $n \rightarrow \infty$ limit as a good approximation, since neutrons at distance $n d_{J}$ give a vanishing contribution for large $n$. Assuming the $n \rightarrow \infty$ limit the effective magnetic fields can be computed straightforwardly. Indeed it is easy to see that the summations in Eqs. (17) and (18) become

$$
\begin{aligned}
\sum_{l=1}^{\infty} C\left(l d_{J}\right)= & \frac{\mathcal{A}}{d_{J}^{2}}\left[\frac{\zeta(3)}{d_{J}}-\frac{\mathcal{B}}{d_{J}} \operatorname{Li}_{3}\left(e^{-m d_{J}}\right)-\mathcal{B} m \operatorname{Li}_{2}\left(e^{-m d_{J}}\right)\right] ; \\
\sum_{l=1}^{\infty} D\left(l d_{J}\right)= & \frac{3 \mathcal{A}}{d_{J}^{3}} \zeta(3)+\frac{\mathcal{A B} m^{2}}{d_{J}} \log \left(1-e^{-m d_{J}}\right) \\
& -\frac{3 \mathcal{A B} m}{d_{J}^{2}} \operatorname{Li}_{2}\left(e^{-m d_{J}}\right)-\frac{3 \mathcal{A B}}{d_{J}^{3}} \operatorname{Li}_{3}\left(e^{-m d_{J}}\right),
\end{aligned}
$$

where $J=I, I I, \zeta(s)$ stands for the Riemann zeta function while $\operatorname{Li}_{s}(z)=\sum_{n=1}^{\infty} \frac{z^{n}}{n^{s}}$ is the Polylogarythm function [48]. Exploiting these results the effective magnetic fields can thus be written as $\left(\mu \boldsymbol{B}_{J}=\mu B_{J} \boldsymbol{P}_{J}\right.$, with $\left.J=I, I I\right)$

$$
\begin{aligned}
\mu B_{I}= & -\frac{2 \mathcal{A} \zeta(3)}{d_{I}^{3}}+\frac{2 \mathcal{A B}}{d_{I}^{3}} \operatorname{Li}_{3}\left(e^{-m d_{I}}\right)+\frac{2 \mathcal{A B} m}{d_{I}^{2}} \operatorname{Li}_{2}\left(e^{-m d_{I}}\right) \\
\mu B_{I I}= & \frac{4 \mathcal{A} \zeta(3)}{d_{I I}^{3}}-\frac{4 \mathcal{A B}}{d_{I I}^{3}} \operatorname{Li}_{3}\left(e^{-m d_{I I}}\right) \\
& -\frac{4 \mathcal{A B} m}{d_{I I}^{2}} \operatorname{Li}_{2}\left(e^{-m d_{I I}}\right)+\frac{2 \mathcal{A B} m^{2}}{d_{I I}} \log \left(1-e^{-m d_{I I}}\right)
\end{aligned}
$$

\subsection{Evolution of the neutron state and phase difference}

The evolution of the neutron state $\psi_{J}$, for both subbeams $(J=I, I I)$, is governed by the Schroedinger equation

$i \partial_{t} \psi_{J}=\left(-\frac{\nabla^{2}}{2 M}+M\right) \psi_{J}-\boldsymbol{\sigma} \cdot\left[\mu\left(\boldsymbol{B}_{J}+\boldsymbol{B}_{J}^{0}\right)\right] \psi_{J}$

where $\psi_{J}$ is the product of a spatial wave-function (a plane wave $f(t) e^{i \boldsymbol{k} \cdot \boldsymbol{x}}$ if the beam is perfectly monochromatic) and a spin function. It is convenient to write the spinor for each subbeam in the basis defined by the corresponding polarization, namely $\boldsymbol{\sigma} \cdot \boldsymbol{P}_{J}|\uparrow J\rangle=|\uparrow J\rangle$ and $\boldsymbol{\sigma} \cdot \boldsymbol{P}_{J}\left|\downarrow_{J}\right\rangle=-\left|\downarrow_{J}\right\rangle$.

It is now appropriate that we discuss to what extent the single particle description suits the evolution of the neutron state. The many-neutron state corresponding to the single particle states $|\uparrow J\rangle$ is

$$
|J\rangle=\prod_{k}|\uparrow J, k\rangle
$$

where the product extends over all the neutrons of the subbeam. The interaction Hamiltonian of Eq. (13) for the neutron subbeams can be written as

$H=\frac{1}{2} \sum_{u, v} \sum_{l \neq m} K^{u v}\left((l-m) d_{J} \hat{\boldsymbol{y}}\right) \sigma_{l}^{u} \sigma_{m}^{v}$,

where $l d_{J}$ specifies the position of the $l$ th neutron along the beam axis and we have taken into account that the nearest neighbour distances are fixed and equal to $d_{J}$ and that all the neutrons are on the beam axis. Employing the definition of Eq. (12), one has

$K^{u v}\left((l-m) d_{J} \hat{\boldsymbol{y}}\right)=C\left((l-m) d_{J}\right) \delta^{u v}-D\left((l-m) d_{J}\right) \delta^{u y} \delta^{v y}$.

Then, from Eq. (13) the interaction Hamiltonian becomes

$$
\begin{aligned}
H= & \frac{1}{2} \sum_{l \neq m}\left[C\left((l-m) d_{J}\right)\left(\sigma_{l}^{x} \sigma_{m}^{x}+\sigma_{l}^{z} \sigma_{m}^{z}\right)\right. \\
& \left.+\left(C\left((l-m) d_{J}\right)-D\left((l-m) d_{J}\right)\right) \sigma_{l}^{y} \sigma_{m}^{y}\right] .
\end{aligned}
$$

Introducing the couplings $F_{l m}^{x J}=C\left((l-m) d_{J}\right)$ and $F_{l m}^{y J}=$ $\left(C\left((l-m) d_{J}\right)-D\left((l-m) d_{J}\right)\right)$, we can write more compactly

$$
H=\frac{1}{2} \sum_{l \neq m}\left[F_{l m}^{x J}\left(\sigma_{l}^{x} \sigma_{m}^{x}+\sigma_{l}^{z} \sigma_{m}^{z}\right)+F_{l m}^{y J} \sigma_{l}^{y} \sigma_{m}^{y}\right] .
$$

To the axion-mediated interaction we must add the term due to the external magnetic field. For the two subbeams we have respectively

$H_{B}^{I}=-\mu B_{0} \sum_{l} \sigma_{l}^{z} ; \quad H_{B}^{I I}=-\mu B_{0} \sum_{l} \sigma_{l}^{y}$.

Without loss of generality we have assumed that the external magnetic field for the subbeam I is along the $z$ axis. The total Hamiltonian of subbeam II $H^{I I}=H+H_{B}^{I I}$ is formally equivalent to that of the $X X Z$ chain. It is clear that the fully magnetized state $|I I\rangle$ of Eq. (22) is an eigenstate of $H^{I I}$ (actually the ground state). As a consequence the total magnetization and the total angular momentum are conserved, since

$\left\langle I I\left|\left[H^{I I}, S^{u}\right]\right| I I\right\rangle=0$

for each $u=x, y, z$ and $S^{u}=\sum_{l} \sigma_{l}^{u}$. For the first subbeam the fully magnetized state $|I\rangle$ is not an exact eigenstate of the total Hamiltonian $H^{I}=H+H_{B}^{I}$. However the term due to 
the external magnetic field dominates on the spin-spin interaction term. To a very good approximation the fully magnetized state is the ground state of the many-body Hamiltonian. Therefore, also for subbeam $I$, the total magnetization and the total angular momentum are conserved. Since the ground state of subbeam II is $|I I\rangle$ and the ground state of subbeam I is $|I\rangle$, and assuming that the two subbeams are found in these states, it is reasonable to assume that each neutron of the $J$ th subbeam is in the single particle state $|\uparrow J\rangle$. This justifies the single particle description of the neutron state evolution. Clearly this constitutes an approximation, and the full many-body dynamics should be taken into account for a more realistic description. This will be analyzed in a forthcoming paper.

We assume that as soon as the neutron leaves BS, it is found in the up state for the corresponding sub-beam; at this instant we set $t=0$. If $y$ denotes the coordinate along the propagation axis, with $y=0$ at the beginning of the optical path, thereby setting $t=0$, we have

$\psi_{J}(t)=f(t) e^{i k y}|\uparrow J\rangle$,

where $f(t)$ is a function to be determined. Assuming $f(t)=$ $e^{-i \omega_{J} t}$ and substituting in Eq. (21) gives

$\omega_{J}=\frac{k^{2}}{2 M}+M-\mu B_{J}-\mu B_{0}$.

Then the total phase accumulated in a time $t$ for a neutron in subbeam $J$ is equal to

$$
\begin{aligned}
\phi_{J}(t) & =\arg \left(\left\langle\psi_{J}(0) \mid \psi_{J}(t)\right\rangle\right) \\
& =-\left(\frac{k^{2}}{2 M}+M-\mu\left(B_{J}+\mu B_{0}\right)\right) t .
\end{aligned}
$$

It follows that the phase difference between the two subbeams at the interference plane is, after a time $t$,

$\Delta \phi(t)=\phi_{I I}(t)-\phi_{I}(t)=\mu\left(B_{I I}-B_{I}\right) t$

which can be computed immediately with the aid of Eq. (20). Assuming $d_{I}=d_{I I}=d$, the phase difference can be compactly expressed as

$\Delta \phi(t)=\left[G_{m}(d)+G_{a}(d)\right] t$,

where $G_{m}(d)$ results from the dipole-dipole interaction and $G_{a}(d)$ comes from the axion-mediated interaction. These are

$$
\begin{aligned}
G_{m}(d)= & \frac{6 \mathcal{A}}{d^{3}} \zeta(3) \\
G_{a}(d)= & -\frac{6 \mathcal{A B}}{d^{3}} \operatorname{Li}_{3}\left(e^{-m d}\right)-\frac{6 \mathcal{A B} m}{d^{2}} \operatorname{Li}_{2}\left(e^{-m d}\right) \\
& +\frac{2 \mathcal{A B} m^{2}}{d} \log \left(1-e^{-m d}\right) .
\end{aligned}
$$

To the phase difference $\Delta \phi(t)$ one must in principle add a possible phase shift $\Delta \phi_{B S}$ due to the operations conducted in the beam splitter and in the Stern-Gerlach like apparatus, which for our purposes should be as small as possible.

To isolate the axion contribution in the phase difference, one can set the beam path (and then the evolution time $t$ ) in such a way that the first term, due to dipole-dipole interactions, is an integer multiple of $2 \pi$, since this phase difference is indistinguishable from a vanishing phase difference. These times, for each integer $k$, are clearly given by ${ }^{1}$ $T_{k}=\frac{2 k \pi}{G_{m}(d)}=\frac{k \pi d^{3}}{3 \mathcal{A} \zeta(3)}$, and the phase difference, evaluated at $T_{k}$, reads

$$
\begin{aligned}
\Delta \phi\left(T_{k}\right)= & \left\{\frac { k \pi \mathcal { B } } { 3 \zeta ( 3 ) } \left[2 m^{2} d^{2} \log \left(1-e^{-m d}\right)\right.\right. \\
& \left.\left.-6 m d \operatorname{Li}_{2}\left(e^{-m d}\right)-6 \operatorname{Li}_{3}\left(e^{-m d}\right)\right]\right\}_{\bmod 2 \pi} .
\end{aligned}
$$

Notice that this phase is non-zero only in presence of ALPs, since it vanishes for $\mathcal{B}=0$ (recall that $\mathcal{B}=\frac{g_{a N N}^{2}}{\pi \alpha g^{2}}$ ). It is instructive to study the massless limit of equation (34). For $m \rightarrow 0$ it reduces to $\left(\operatorname{Li}_{3}(1)=\zeta(3)\right)$

$\Delta \phi\left(T_{k}\right)=\left\{-\frac{2 k \pi \mathcal{B}}{\zeta(3)} \operatorname{Li}_{3}(1)\right\}_{\bmod 2 \pi}=\{-2 k \pi \mathcal{B}\}_{\bmod 2 \pi}$.

From this equation we can see that the distance dependence disappears when $m \rightarrow 0$, and the phase difference depends only on the axion-neutron coupling $g_{a N N}$ through $\mathcal{B}$. Notice that Eq. (35) holds to a very good accuracy for the ultra-light axions [20], which have masses of the order $m \sim 10^{-22} \mathrm{eV}$.

The pseudoscalar neutron-neutron interaction (1) is not necessarily originated by ALP fields. Any new pseudoscalar field that interacts with standard model fermions via a Lagrangian of the kind of Eq. (1) in principle affects the neutron phase in the same way as in Eq. (34). Clearly when other fields are involved, the coupling constant $g_{a j}$, and consequently $\mathcal{B}$ have to be changed accordingly.

\section{Numerical analysis}

In this section we provide a numerical analysis of the phase difference and discuss the main results. As it is evident from Eq. (34), the phase difference, evaluated at the $k$ th recurrence time $T_{k}$, is proportional to the parameter $\mathcal{B} \propto g_{p}^{2}$. In the range of ALP masses $m \in\left[10^{-6}-1\right] \mathrm{eV}$, and inter-neutron distances $d \in\left[10^{-11}-10^{6}\right] \mathrm{m}$, the phase depends only weakly on the parameters $m, d$, while keeping a relatively strong dependence on the coupling.

In Fig. 2 we plot the phase difference, modulo $2 \pi$, evaluated at the minimum recurrence time $T_{1}$ for several values of

\footnotetext{
1 The knowledge of these istants is limited by the uncertainties on the magnetic dipole moment of the neutrons, because of the $\mathcal{A}$ factor. We remark that this kind of procedure is different from an a priori cancellation of the standard magnetic interaction.
} 


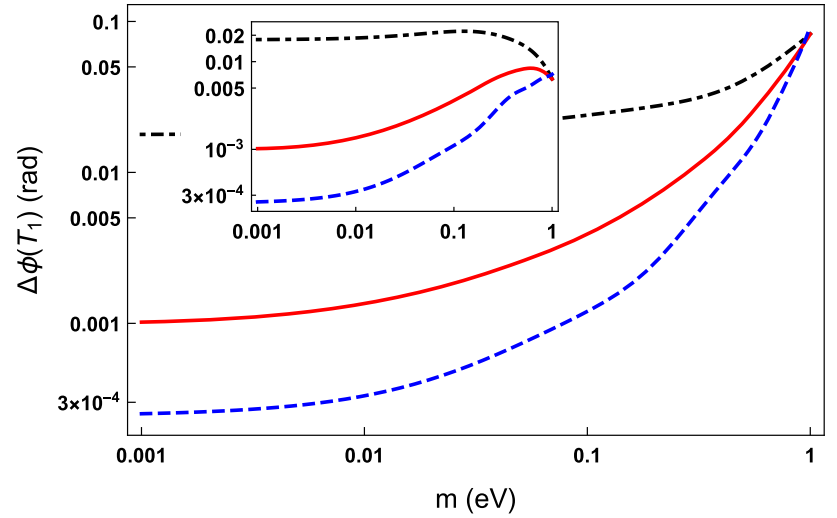

Fig. 2 Logarithmic scale plot of the phase difference $\left|\Delta \phi\left(T_{1}\right)\right|$ (Eq. (34)) modulo $2 \pi$ for several values of the coupling constant in the ALP mass range $\left[10^{-3}, 1\right] \mathrm{eV}$ for an inter-neutron distance $d=10^{-8} \mathrm{~m}$ ( $d=10^{-6} \mathrm{~m}$ in the inset). In particular: (black dot-dashed line) $g_{a N N}=g_{C P}$, where $g_{C P}$ is the threshold from effective Casimir pressure measurements [33] and sample values are $g_{C P}=0.0327$ for $m=10^{-3} \mathrm{eV}, g_{C P}=0.0348$ for $m=0.05 \mathrm{eV}, g_{C P}=0.0674$ for $m=1 \mathrm{eV}$; (red solid line) $g_{a N N}=g_{C F}$, where $g_{C F}$ is the threshold from measurements of the difference of Casimir forces [35] and sample values are $g_{C F}=0.007$ for $m=10^{-3} \mathrm{eV}, g_{C F}=0.012$ for $m=0.05 \mathrm{eV}, g_{C F}=0.066$ for $m=1 \mathrm{eV}$; (blue dashed line) $g_{a N N}=g_{I E}$, where $g_{I E}$ is the threshold from isoelectronic experiments [34], and sample values are $g_{I E}=0.0036$ for $m=10^{-3} \mathrm{eV}$, $g_{I E}=0.006$ for $m=0.05 \mathrm{eV}, g_{I E}=0.07$ for $m=1 \mathrm{eV}$

the coupling constant $g_{a N N}$ in the mass range $\left[10^{-3}, 1\right] \mathrm{eV}$. The couplings are chosen compatibly with the modelindependent constraints from several experimental analyses [33-35]. For $m<0.1 \mathrm{eV}$ we see that the phase difference is essentially the same for $d=10^{-8} \mathrm{~m}$ and $d=10^{-6} \mathrm{~m}$, showing that the dependence on the distance becomes relevant only when the product $m d$ is quite high [right tail of the curves in the inset of (2)]. This obviously traces back to the Yukawa damping factor $e^{-m d}$ which accompanies the axionmediated interaction, and can be clearly seen from Eq. (34) when $m d \rightarrow 0: \Delta \phi\left(T_{k}\right) \simeq[-2 k \pi \mathcal{B}]_{\bmod 2 \pi}$, implying that when $m d \ll 1$, the phase difference essentially depends only upon the coupling $g_{a N N}$ (via the parameter $\mathcal{B}$ ).

A similar conclusion can be drawn from Fig. 3. Here the damping is evident for $d=10^{-6} \mathrm{~m}$ (lower panel). Figure 3 also shows clearly the $g_{a N N}^{2}$ dependence of the phase difference and an extremely weak dependence on the ALP mass in the ranges considered.

In the massless limit the phase difference no longer depends on $d$ (see Eq. (35)) and this allows to choose, in principle, an arbitrarily large inter-neutron distance. Consequently, many of the limitations that are present in the massive case and that affect the implementation of our setup, disappear in the massless case. The results presented in the figure are in the spirit of a model-independent analysis, which in principle applies to any ALP which experiences an interaction with neutrons. Since masses and couplings vary sensibly
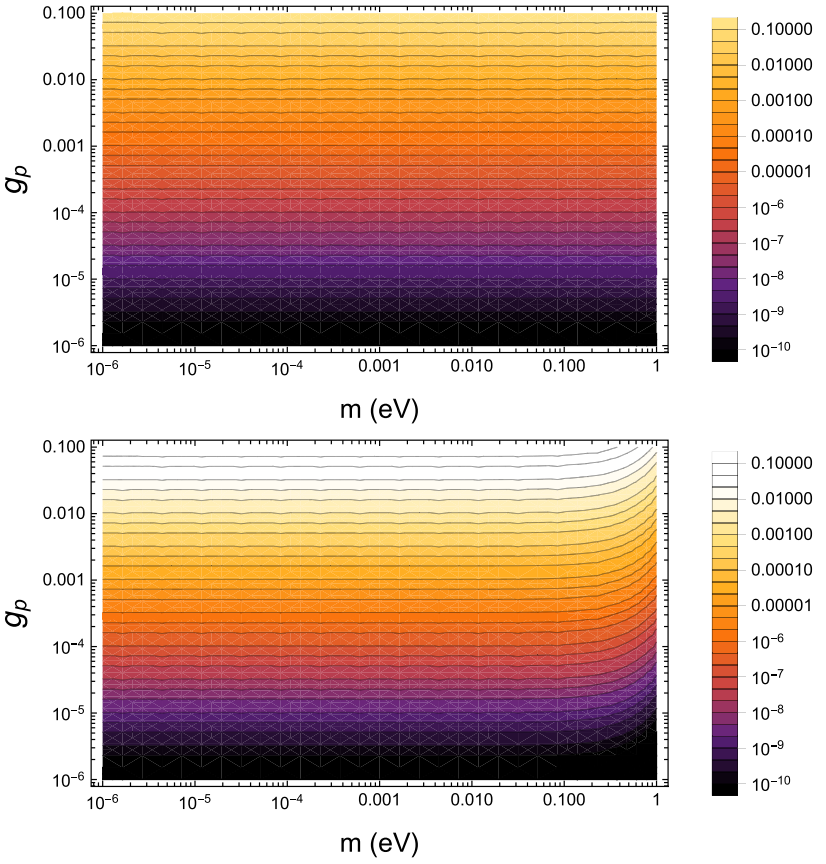

Fig. 3 Contour plots of the phase difference $\left|\Delta \phi\left(T_{1}\right)\right|$, modulo $2 \pi$, in the mass-coupling plane for $d=10^{-9} \mathrm{~m}$ (upper panel) and $d=10^{-6} \mathrm{~m}$ (lower panel) in the range $\left(m, g_{a N N}\right) \in\left[10^{-6}, 1\right] \mathrm{eV} \times\left[10^{-6}, 10^{-1}\right]$. The Yukawa damping is visible in the lower panel at the right end of the plot, where curves corresponding to lower values of the phase difference are pushed up vertically

from a model to another, we choose a relatively wide range of parameters, with $\left(m, g_{a N N}\right) \in\left[10^{-6}, 1\right] \mathrm{eV} \times\left[10^{-6}, 10^{-1}\right]$.

Notice that the current laboratory constraints on $g_{a N N}$ from fifth force experiments [33-36,45,46] set an upper bound of the order $\sim 10^{-4}$. Our setup could in principle improve on such bound. We stress, however, that at this stage our proposal is theoretical and a more realistic analysis is demanded to a forthcoming paper.

For inter-neutron distances $d<1 \mu \mathrm{m}$ the phase difference is essentially independent on the ALP mass, being sensible to the coupling constant alone. This means that while the mass range considered can be enlarged to include lower and lower masses without affecting the results, the possibility to measure the phase difference at lower coupling constants is limited by the sensibility achievable in the experiment.

It should be remarked that when the inter-neutron distance $d$ is small enough, one can evaluate the phase difference at the $k$ th recurrence time with a high $k$, while keeping the propagation time reasonable. This has the effect of bringing along a multiplicative $k$ factor in the phase difference, see Eq. (34), which in principle may render it observable at lower values of the coupling constant. For instance, at $d=10^{-9} \mathrm{~m}$ one can choose $k \simeq 4000$ while still keeping $T_{k}<1$ s.

The observability of the phase difference is limited by the finite precision achievable in the experiment, and from the elements over which one has only a poor control. The 
first obvious limitation comes from the need for a recurrence time $T$ in order to isolate the axion contribution to the phase difference. This time cannot be arbitrarily long, because of the finite neutron lifetime $\tau_{n} \simeq 880$ s and especially because of a finite coherence length (and therefore a finite coherence time) which may be the result of unwanted interactions with the environment. Recurrence times of the order of the second can be obtained for $d \simeq 10^{-8} \mathrm{~m}$.

At the opposite end stands the issue of time resolution. A recurrence time too small would imply an extremely fast oscillation of the phase difference, rendering its analysis uneasy. This is not really a problem, unless very small interneutron distances $d<10^{-11} \mathrm{~m}$ are considered. The minimum recurrence time $T$ also has an obvious impact on the size of the interferometric apparatus needed. If $v$ denotes the average neutron velocity, the arm length must be at least $L=v T$. If $T \simeq$ s and $L$ must be of the order of meters, the average neutron velocity cannot exceed a few $\mathrm{m} / \mathrm{s}$. For this reason ultra cold neutrons are to be preferred, as they can have velocities as small as $5 \mathrm{~m} / \mathrm{s}$ [49]. The second important limitation comes from the total neutron flux available, and therefore the available beam intensity $I$. Because of the several devices involved in the preparation of the beam (collimator, monochromator and so on) the flux loss may be significant. A relatively high intensity of the beam, on the other hand, is essential for a sufficiently small inter-neutron distance. In turn, a small $d$ guarantees a reasonable recurrence time. If the neutron velocity is of the order of $v \simeq 1 \mathrm{~m} / \mathrm{s}$, a distance $d \simeq 10^{-8} \mathrm{~m}$ is obtained in correspondence with an intensity $I \simeq 10^{8} \mathrm{n} / \mathrm{s}$. This is reasonable, given that pulsed neutron beams can reach intensities of the order $I \simeq 10^{10}$ $\mathrm{n} / \mathrm{s}[50]$.

\subsection{The parameter space}

Here we briefly discuss our choice of parameters. We point out that the parameter space we consider is ruled out by astrophysical arguments but is compatible with the theoretical analyses on the Casimir effect and fifth force experiments [33-35,51]. We stress that the astrophysical bounds are strongly model dependent and rely on a great deal of assumptions on the underlying physics [42-44]. The range of parameters that we consider is the one typical of fifth force experiments.

We remark that the ALPs we consider are not necessarily QCD Axions but pseudoscalar particles which have properties similar to the axion and that the relation $g_{a f}=$ $\left(C_{a f} m_{f}\right) / f_{a}$ which holds for QCD axions is not necessarily verified for a generic ALP, like those we are analyzing. Therefore no conclusion on $f_{a}$ and on the relation $g_{a f}=\left(C_{a f} m_{f}\right) / f_{a}$ can a priori be drawn.

Collider experiments study the existence of heavy ALPs with $m>100 \mathrm{MeV}$ [52,53]. Our proposal is not appropriate for this kind of ALPs, since the phase difference in equation (34) is exponentially suppressed, independently of the coupling constant, because of the Yukawa factor $e^{-m d}$, even for small distances.

The constraints on axion-like particles from pion decays are relevant for axion masses of the order of $10-100 \mathrm{MeV}$ [54]. These values lie outside the range of masses that we can probe. Moreover this kind of experiments is sensitive to the axion-photon coupling and the meson-axion mixing angle but give no direct constraint on the axion-nucleon couplings.

We point out that the experiments (Light shining through a wall [55], CAST [55], SUMICO [55], SN1987a [56], LEP [57], LHC [58,59], Meson decays [54]), study the axionphoton interaction and constrain the related coupling constant $g_{a \gamma \gamma}$ but provide no direct and model independent constraints on $g_{a N N}$. The strategy we propose, which instead focuses on the fermion-fermion interaction mediated by the ALPs, may allow to cover a new parameter space, and eventually test some ALP models, like those considered in the references [33-35,51].

\section{Discussions and conclusions}

In conclusion, we have shown that the fermion-fermion interaction mediated by axions produces a non trivial contribution to the total phase due to the time evolution of neutrons that can be exploited to detect the existence of such elusive particles. This can be done using standard interferometric techniques. Indeed, if two neutron beams travel in the two arms of an interferometer, and the distances among the neutrons are sufficiently large, the interference between the two beams depends only on the magnetic and axion-mediated interactions. In our idealized experiment we consider the simplest geometric configuration and inter-neutron distances which cannot be obtained in the present experiments. This is done in order to describe our theoretical proposal.

A forthcoming paper will be devoted to a more complex and realistic configuration, considering a cylindrical geometry for the neutron beam and employing wave packets for neutron propagation, in order to make our proposal implementable in future experiments. Limited to the theoretical configuration we have considered, we have shown that one can have a contribution to the phase difference for the dipoledipole interaction which is an integer multiple of $2 \pi$ and then obtain a phase difference entirely due to the neutron-neutron interaction induced by axions.

By considering ultra cold neutrons, we derived values of phase differences which are in principle experimentally detectable for a wide range of ALP parameters. The theoretical results obtained for $\Delta \phi\left(T_{1}\right)$ are indeed compatible with the sensitivity reached in neutron interferometry [37]. Therefore, our results, show that future experiments on neu- 
tron interferometry could be used to analyze the interaction induced by axions and to verify the existence of some types of ALPs, thus opening new horizons in the study of such elusive particles. In our treatment, for simplicity, we have modelled the collimated cold neutron beam as a one-dimensional chain of neutrons which translates rigidly. We neglected the losses due to the propagation, and considered the beam intensity as a constant. These approximations shall be improved upon in future works.

Acknowledgements A.C. and A.Q thank partial financial support from Ministero dell'Università e della Ricerca (MUR) and Istituto Nazionale di Fisica Nucleare (INFN). A.C. also thanks the COST Action CA1511 Cosmology and Astrophysics Network for Theoretical Advances and Training Actions (CANTATA). SMG also acknowledges the QuantiXLie Center of Excellence (Grant KK.01.1.1.01.0004)

Data Availability Statement This manuscript has no associated data or the data will not be deposited. [Authors' comment: The paper is selfcontained and does not necessitate any additional data as figures, tables or plots.]

Open Access This article is licensed under a Creative Commons Attribution 4.0 International License, which permits use, sharing, adaptation, distribution and reproduction in any medium or format, as long as you give appropriate credit to the original author(s) and the source, provide a link to the Creative Commons licence, and indicate if changes were made. The images or other third party material in this article are included in the article's Creative Commons licence, unless indicated otherwise in a credit line to the material. If material is not included in the article's Creative Commons licence and your intended use is not permitted by statutory regulation or exceeds the permitted use, you will need to obtain permission directly from the copyright holder. To view a copy of this licence, visit http://creativecomm ons.org/licenses/by/4.0/.

Funded by SCOAP ${ }^{3}$.

\section{References}

1. J. Ellis, Nucl. Phys. A 827, 187-198 (2009)

2. S.M. Bilenky, B. Pontecorvo, Phys. Lett. B 61, 248 (1976)

3. S.M. Bilenky, B. Pontecorvo, Yad. Fiz. 3, 603 (1976)

4. O. Nachtmann, Elementary Particle Physics: Concepts and Phenomena (Springer, Berlin, 1990)

5. A. Capolupo, G. Lambiase, A. Quaranta, Phys. Rev. D 101, 095022 (2020)

6. V. Rubin, W.K. Thonnard Jr., N. Ford, Astrophys. J. 238, 471 (1980)

7. N. Aghanim et al., Planck Collaboration, Astron. Astrophys. 641, A6 (2020)

8. R.D. Peccei, H. Quinn, Phys. Rev. Lett. 38, 1440 (1977)

9. R.D. Peccei, H. Quinn, Phys. Rev. D 16, 1791 (1977)

10. S. Weinberg, Phys. Rev. Lett. 40, 223 (1978)

11. F. Wilczek, Phys. Rev. Lett. 40, 279 (1978)

12. G.G. Raffelt, J. Phys. A 40, 6607 (2007)

13. D.J.E. Marsch, Phys. Rep 643, 1 (2016)

14. M. Dine, W. Fischler, M. Srednicki, Phys. Lett. B 104, 199 (1981)

15. A. Zhitnitsky, Sov. J. Nucl. Phys. 31, 260 (1980)

16. Particle Data Group P. A. Zyla et al., Progress of Theoretical and Experimental Physics, vol. 2020, Issue 8, August 2020, 083C01 (2020)

17. J.E. Kim, Phys. Rev. Lett. 43, 103 (1979)
18. M.A. Shifman, A. Vainshtein, V.I. Zakharov, Nucl. Phys. B 1(66), $493(1980)$

19. J.E. Kim, D.J.E. Marsch, Phys. Rev. D 93, 025027 (2016)

20. I. De Martino, T. Broadhurst, S.-H. Henry-Tye, T. Chiueh, H.-Y. Schive, R. Lazkoz, Phys. Rev. Lett. 119, 221103 (2017)

21. V.A. Rubakov, JETP Lett. 65, 621-624 (1997)

22. E. Zavattini et al., PVLAS Collaboration, Phys. Rev. D 77, 032006 (2008)

23. P. Pugnat et al. (OSQAR Collaboration), Phys. Rev. D 78, 092003 (2008)

24. R. Ballou et al. (OSQAR Collaboration), Phys. Rev. D 92, 092002 (2015)

25. K. Ehret et al. (ALPS Collaboration), Phys. Lett. B 689(4-5), 149155 (2010)

26. S. Aune et al. (CAST Collaboration), Phys. Rev. Lett. 107, 261302 (2011)

27. N. Du et al. (ADMX Collaboration), Phys. Rev. Lett. 120, 151301 (2018)

28. R. Barbieri et al., Phys. Dark Univ. 15, 135-141 (2017)

29. A. Capolupo, G. Lambiase, G. Vitiello, Adv. High Energy Phys. $826051(2015)$

30. A. Capolupo, I. De Martino, G. Lambiase, A. Stabile, Phys. Lett. B 790, 427-435 (2019)

31. J.E. Moody, F. Wilczek, Phys. Rev. D 30, 130 (1984)

32. R. Daido, F. Takahashi, Phys. Lett. B 772, 127 (2017)

33. V.B. Bezerra, G.L. Klimchitskaya, V.M. Mostepanenko, C. Romero, Eur. Phys. J. C 74, 2859 (2014)

34. G.L. Klimchitskaya, V.M. Mostepanenko, Eur. Phys. J. C 75, 164 (2015)

35. G.L. Klimchitskaya, V.M. Mostepanenko, Phys. Rev. D 95, 123013 (2017)

36. A. Capolupo, S.M. Giampaolo, G. Lambiase, A. Quaranta, Phys. Lett. B 804, 135407 (2020)

37. H. Rauch, S.A. Werner, Neutron Interferometry-Lessons in Experimental Quantum Mechanics, Wave-Particle Duality, and Entanglement, 2nd edn. (Oxford University Press, Oxford, 2015)

38. S.A. Werner, J.-L. Staudenmann, R. Colella, Phys. Rev. Lett. 42, 1103 (1979)

39. B.E. Allman, H. Kaiser, S.A. Werner, A.G. Wagh, V.C. Rakhecha, J. Summhammer, Phys. Rev. A 56, 4420 (1997)

40. A.G. Wagh, V.C. Rakhecha, Phys. Lett. A 148(1-2), 17-19 (1990)

41. A.G. Wagh, V.C. Rakhecha, P. Fischer, A. Ioffe, Phys. Rev. Lett. 81, 1992 (1998)

42. L.B. Leinson, JCAP09, 2021, 001 (2021)

43. A. Sedrakian, Phys. Rev. D 93, 065044 (2016)

44. A. Sedrakian, Phys. Rev. D 99, 043011 (2019)

45. C.A.J. O'Hare, E. Vitagliano, Phys. Rev. D 102, 115026 (2020)

46. L. Chen, J. Liu, K. Zhu, Constraining Axion-to-Nucleon interaction via ultranarrow linewidth in the Casimir-less regime (2021). arXiv:2107.08216 [quant-ph]

47. F. Ott, S. Kozhevnikov, A. Thiaville, J. Torrejon, M. Vazquez, Nucl. Instrum. Methods Phys. Res. A 788, 29-34 (2015)

48. I.S. Gradshteyn, I.M. Ryzhik, Table of Integrals, Series and Products, 7th edn. (Academic Press, Cambridge, 2007), pp. 110-111

49. A. Steyerl, Phys. Lett. B 29(1), 33-35 (1969)

50. International Atomic Energy Agency, Compendium of Neutron Beam Facilities for High Precision Nuclear Data Measurements, IAEA-TECDOC-1743, IAEA, Vienna (2014)

51. G.L. Klimchitskaya, P. Kuusk, V.M. Mostepanenko, Phys. Rev. D 101, 056013 (2020)

52. J. Jaeckel, M. Spannowsky, Phys. Lett. B 753, 482-487 (2016)

53. M. Bauer, M. Neubert, A. Thamm, J. High Energy Phys. 2017, 44 (2017)

54. W. Altmannhofer, S. Gori, D.J. Robinson, Phys. Rev. D 101, 075002 (2020) 
55. P.W. Graham, I.G. Irastorza, S.K. Lamoreaux, A. Lindner, K.A. van Bibber, Ann. Rev. Nucl. Part. Sci. 65, 485-514 (2015)

56. J.H. Chang, R. Essig, S. McDermott, J. High Energy Phys. 2018, $51(2018)$

57. O.P.A.L. Collaboration, G. Abbiendi et al., Eur. Phys. J. C 26, 331344 (2003)
58. S. Knapen, T. Lin, H.K. Lou, T. Melia, Phys. Rev. Lett. 118, 171801 (2017)

59. M. Bauer, M. Heiles, M. Neubert, A. Thamm, Eur. Phys. J. C 79, 74 (2019) 